\title{
A Model Experiment for Piezoelectric Relaxations in Polymers
}

\author{
Hiroyuki Yamagami and Eiichi Fukada \\ The Institute of Physical and Chemical \\ Research, Wako, Saitama, Japan.
}

(Received May 29, 1973)

\begin{abstract}
Based on mechanical and electrical three-element models for piezoelectric polymers, mathematical expressions for the piezoelectric stress-constant, $e$, and strain-constant, $d$, are derived as functions of the elastic and dielectric constants in a piezoelectric crystalline phase and a nonpiezoelectric amorphous phase, and the volume fraction of the piezoelectric phase. If no relaxations exist in the piezoefectric phase, apparent piezoelectric relaxations are caused only by elastic and dielectric relaxations in the amorphous phase.

Model experiments for a two-phase polymer are made using oriented silk fibers as the piezoelectric phase and an adhesive layer of epoxy resin or poly(vinyl alcohol) as the amorphous phase. In the epoxy resin case the elastic relaxation prevails over the dielectric relaxation, which accounts for the decrease of $e$-constant and the increase of $d$-constant with increasing temperature observed for the silk-epoxy resin system. In the poly(vinyl alcohol) case the dielectric relaxation dominates the elastic relaxation, which explains the increase of both $e$ - and $d$-constants with the increase of temperature for the silk-poly(vinyl alcohol) system.

KEY WORDS Piezoelectricity / Relaxation / Viscoelasticity / Dielectricity / Three-Element Model / Silk / Epoxy Resin / Poly(vinyl alcohol)/
\end{abstract}

The piezoelectricity of polymers has been studied by many workers. ${ }^{1}$ The piezoelectric constants of polymers depend markedly upon the temperature and frequency of measurement in a similar way to the elastic and dielectric constants. One of the current problems in the study of polymer piezoelectricity is to clarify the mechanisms of this piezoelectric relaxation.

Recently theoretical studies for piezoelectric relaxations in polymers have been published by Hayakawa and Wada., ${ }^{2,3}$ A theory of a spherical dispersion model has been also presented. by Date. ${ }^{4}$

Most piezoelectric polymers consist of piezoelectric crystals and a nonpiezoelectric amorphous phase and the crystallites are dispersed in the amorphous phase. The piezoelectric effect may take place not only in the crystalline phase but also in regions where the macromolecules are well oriented. In the following description such oriented phases are regarded as being included in the crystalline phase. It has been experimentally established that piezoelectric relaxations in polymers are closely associated with the elastic and dielectric relaxations.

If we assume that piezoelectric crystals show no elastic and dielectric relaxations and that the amorphous phases surrounding crystals show elastic and dielectric relaxations, we can anticipate that the piezoelectric constants observable for the entire system will also display a relaxation. In this paper we have performed some model experiments by employing silk fibers as a piezoelectric crystalline phase and either poly(vinyl alcohol) (PVA) or epoxy resin as a nonpiezoelectric amorphous phase.

\section{THEORETICAL EQUATIONS}

In order to describe the thermodynamical state of a piezoelectric system, one can employ four independent variables, strain $S$, stress $T$, electric field $E$, and electric polarization $P$, provided the system is isothermal. In general these variables are expressed as a vector or a tensor.

To simplify the notation we consider a onedimensional piezoelectric effect ignoring anisotropy in the variables. If one chooses a set of 
independent variables $(S, E)$, the corresponding conjugate variables $(T, P)$ are described by the following relations.

$$
\begin{aligned}
& P=e S+\eta^{S} E \\
& T=G^{E} S-e E
\end{aligned}
$$

If the independent variables are changed to $T$ and $E$, we obtain,

$$
\begin{aligned}
& P=d T+\eta^{T} E \\
& S=J^{E} T+d E
\end{aligned}
$$

where $e$ is the piezoelectric stress-constant, $d$ the piezoelectric strain-constant, $\eta^{S}$ and $\eta^{T}$ the electric susceptibility at $S=0$ and $T=0$, respectively, $G^{E}$ the elastic constant at $E=0$, and $J^{E}$ the elastic compliance at $E=0$. As will be described below, we determine the values of $e, d$ and $G^{E}$ under the condition of $E=0$.

Now we wish to derive the expressions for these constants based on a simple model shown in Figures 1 and 2. In Figure 1 we represent
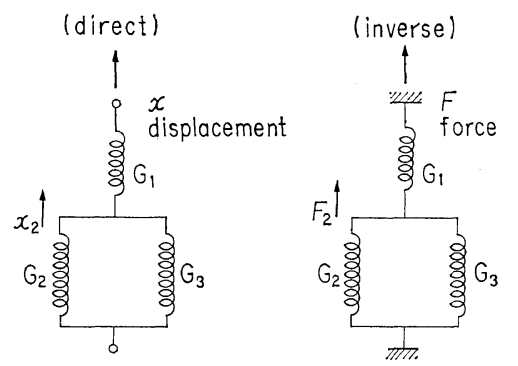

Figure 1. Mechanical model for a two-phase piezoelectric polymer.

the piezoelectric phase by a spring whose stiffness is $G_{2}$. The nonpiezoelectric phase is given by two springs whose stiffnesses are $G_{1}$ and $G_{3}$ and are connected to the spring $G_{1}$ in series and in parallel, respectively. If we impose an external displacement $x$ on this system, the spring $G_{2}$ deforms by $x_{2}$, which produce a polarization $Q$ on the piezoelectric phase. Mechanical coupling coefficient $k_{\mathrm{m}}$ is thus defined as

$$
k_{\mathrm{m}}=x_{2} / x
$$

By simple calculation $k_{\mathrm{m}}$ is given as follows:

$$
k_{\mathrm{m}}=\frac{G_{1}}{G_{1}+G_{2}+G_{3}}
$$

Figure 2 shows an electrical circuit representing the combination of a condenser $C_{2}$ for the piezoelectric phase and two other condensers $C_{1}$ and $C_{3}$ for the nonpiezoelectric phase connected in series and in parallel with the piezoelectric phase, respectively. Since the measurement of direct piezoelectric effect is performed under the condition where the potential difference between electrodes of the two surfaces is zero, both ends of the circuit in Figure 2 are connected to an earthed short-circuit. The electric charge $Q$ produced by the deformation $x_{2}$ in the piezoelectric phase is divided into three condensers and charge
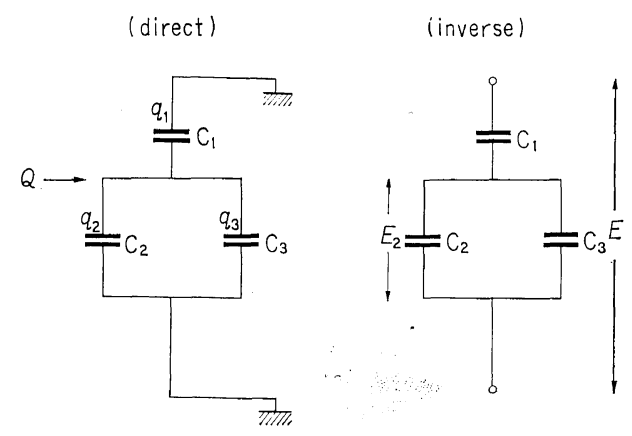

Figure 2. Electrical model for a two-phase piezoelectric polymer.

$q_{1}$ in the condenser $C_{1}$ is actually detected by a charge amplifer connected between two terminals, which are effectively short-circuited. The electrical coupling coefficient $k_{\mathrm{e}}$ is now given by

$$
k_{\mathrm{e}}=q_{1} / Q
$$

which becomes,

$$
k_{\mathrm{e}}=\frac{C_{1}}{C_{1}+C_{2}+C_{3}}
$$

In the case of the inverse piezoelectric effect, we apply a voltage $E$ between two terminals in Figure 2. The ratio of the voltage on the condenser $C_{2}$, which represents the piezoelectric phase, to the applied voltage $E$ is the electrical coupling coefficient $k_{\mathrm{e}}$ and is given by an equation similar to eq 8 .

$$
k_{\mathrm{e}}=\frac{E_{2}}{E}=\frac{C_{1}}{C_{1}+C_{2}+C_{3}}
$$

The force $F_{2}$ is then produced in the piezoelectric phase which is shown by the spring $G_{2}$ 
in Figure 1. For the measurement of inverse piezoelectric effect both ends of the system are mechanically clamped and the force produced by the piezoelectricity is detected. The mechanical coupling coefficient $k_{\mathrm{m}}$ is given by the same equation as eq 6.

$$
k_{\mathrm{m}}=F / F_{2}=\frac{G_{1}}{G_{1}+G_{2}+G_{3}}
$$

The piezoelectric stress-constant $e$ actually observed for the system is proportional to $q_{1} / x$ in the direct effect and $F / E$ in the inverse effect and therefore linearly related to the product of $k_{\mathrm{e}} k_{\mathrm{m}}$.

In order to take into account the dimensional factors in the expression of the e-constant, we make the following assumptions. In Figure 3. the film sample is represented by a rectangular

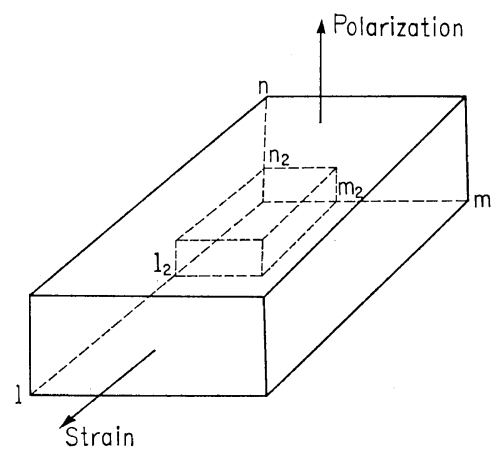

Figure 3. Schematic representation of the crystalline phase $\left(l_{2} \times m_{2} \times n_{2}\right)$ inside the specimen $(l \times$ $m \times n)$.

prism in which the lengths of three sides are $l$, $m$, and $n$. The piezoelectric phase is represented by a rectangular prism with the sides $l_{2}, m_{2}$ and $n_{2}$. The deformation $x$ is given in the direction of $l$ and the polarization is observed in the direction of $n$. Now the apparent piezoelectric stress-constant is given by

$$
e=\frac{q_{1}}{l m} / \frac{x}{l}=\frac{F}{m n} / \frac{E}{n}
$$

and the piezoelectric stress-constant $e_{\mathrm{c}}$ of the piezoelectric phase is given by

$$
e_{\mathrm{c}}=\frac{Q}{l_{2} m_{2}} / \frac{x_{2}}{l_{2}}=\frac{F_{2}}{m_{2} n_{2}} / \frac{E_{2}}{n_{2}}
$$

From eq 11 and 12 we obtain,

$$
\frac{e}{e_{\mathrm{c}}}=\frac{m_{2}}{m} \cdot \frac{q_{1}}{Q} \cdot \frac{x_{2}}{x}=\frac{m_{2}}{m} \cdot \frac{F}{F_{2}} \cdot \frac{E_{2}}{E}
$$

Using eq 8-12, we can obtain, .

$$
e=e_{\mathrm{c}} \frac{m_{2}}{m} k_{\mathrm{m}} k_{\mathrm{e}}=e_{\mathrm{c}} \frac{m_{2}}{m} \cdot \frac{G_{1}}{G_{1}+G_{2}+G_{3}} \cdot \frac{C_{1}}{C_{1}+C_{2}+C_{3}}
$$

It is further transformed to

$$
e=e_{\mathrm{c}} V_{\mathrm{c}} \frac{G_{\mathrm{a}}}{G_{\mathrm{a}}\left(1-V_{\mathrm{c}} \xi\right)+G_{\mathrm{c}} V_{\mathrm{c}} \xi} \cdot \frac{\varepsilon_{\mathrm{a}}}{\varepsilon_{\mathrm{a}}\left(1-V_{\mathrm{c}} \zeta\right)+\varepsilon_{\mathrm{c}} V_{\mathrm{c}} \zeta}
$$

where $V_{\mathrm{c}}=l_{2} m_{2} n_{2} / \mathrm{lmn}$ is the volume fraction of the piezoelectric phase, $\xi=\left(l / l_{2}\right)-1$, and $\zeta=$ $\left(n / n_{2}\right)-1, G_{\text {a }}$ is the elastic constant in the nonpiezoelectric phase represented by the springs $G_{1}$ and $G_{3}, G_{\mathrm{c}}$ the elastic constant in the piezoelectric phase represented by the spring $G_{2}, \varepsilon_{a}$ is the dielectric constant in the nonpiezoelectric phase represented by the capacities $C_{1}$ and $C_{3}$, $\varepsilon_{\mathrm{c}}$ the dielectric constant in the piezoelectric phase represented by the capacity $C_{2}$.

The piezoelectric strain-constant, $d$, is generally given by

$$
d=e / G^{E}
$$

From Figure 1 the stiffness of the whole system is given by

$$
G=\frac{G_{1}\left(G_{2}+G_{3}\right)}{G_{1}+G_{2}+G_{3}}=G^{E} \frac{m n}{l}
$$

From eq 14,16 , and 17 , we get,

$$
d=e_{\mathrm{c}} \frac{m_{2} n}{l} \cdot \frac{l}{G_{2}+G_{3}} \cdot \frac{C_{1}}{C_{1}+C_{2}+C_{3}}
$$

It is further transformed to

$$
\begin{aligned}
d= & e_{\mathrm{c}} V_{\mathrm{c}} \frac{1}{G_{\mathrm{a}}\left[1-V_{\mathrm{c}}(1+\xi)\right]+G_{\mathrm{c}} V_{\mathrm{c}}(1+\xi)} \\
& \times \frac{\varepsilon_{\mathrm{a}}}{\varepsilon_{\mathrm{a}}\left(1-V_{\mathrm{c}} \zeta\right)+\varepsilon_{\mathrm{c}} V_{\mathrm{c}} \zeta}
\end{aligned}
$$

Equations 15 and 19 give the expressions for the $e$-constant and $d$-constant for the two-phase polymer system, which consists of the piezoelectric crystalline phase and the nonpiezoelectric amorphous phase, in terms of the elastic constants and dielectric constants of both phases.

If we assume

$$
\frac{l_{2}}{l}=\frac{m_{2}}{m}=\frac{n_{2}}{n}=\frac{1}{2}
$$


then $V_{\mathrm{c}}=1 / 8, \xi=\zeta=1$ and eq 15 and 19 become,

$$
\begin{aligned}
& e=\frac{1}{8} e_{\mathrm{c}} \cdot \frac{8 G_{\mathrm{a}}}{7 G_{\mathrm{a}}+G_{\mathrm{c}}} \cdot \frac{8 \varepsilon_{\mathrm{a}}}{7 \varepsilon_{\mathrm{a}}+\varepsilon_{\mathrm{c}}} \\
& d=\frac{1}{8} e_{\mathrm{c}} \cdot \frac{8}{6 G_{\mathrm{a}}+2 G_{\mathrm{c}}} \cdot \frac{8 \varepsilon_{\mathrm{a}}}{7 \varepsilon_{\mathrm{a}}+\varepsilon_{\mathrm{c}}}
\end{aligned}
$$

These formula resembles the equations derived by Date $^{4}$ for a spherical dispersion system. For simplicity, we express eq 15 and 19 as follows.

$$
\begin{aligned}
& e=e_{\mathrm{c}} V_{\mathrm{c}} \frac{G_{\mathrm{a}}}{G_{\mathrm{a}}\left(1-K_{G}\right)+G_{\mathrm{c}} K_{G}} \cdot \frac{\varepsilon_{\mathrm{a}}}{\varepsilon_{\mathrm{a}}\left(1-K_{\varepsilon}\right)+\varepsilon_{\mathrm{c}} K_{\varepsilon}} \\
& d=e_{\mathrm{c}} V_{\mathrm{c}} \frac{1}{G_{\mathrm{a}}\left(1-L_{G}\right)+G_{\mathrm{c}} L_{G}} \cdot \frac{\varepsilon_{\mathrm{a}}}{\varepsilon_{\mathrm{a}}\left(1-K_{\varepsilon}\right)+\varepsilon_{\mathrm{c}} K_{\varepsilon}}
\end{aligned}
$$

where $K_{G}=V_{\mathrm{c}} \xi, \quad L_{G}=V_{\mathrm{c}}(1+\xi), \quad$ and $\quad K_{\varepsilon}=V_{\mathrm{c}} \zeta$. Now we assume that the elastic constants and dielectric constants of the two phases are complex quantities as given below:

$$
\begin{aligned}
& G_{\mathrm{a}}=G_{\mathrm{a}}{ }^{\prime}\left[1+j \tan \delta\left(G_{\mathrm{a}}\right)\right] \\
& \boldsymbol{G}_{\mathrm{c}}=G_{\mathrm{c}}{ }^{\prime}\left[1+j \tan \delta\left(\boldsymbol{G}_{\mathrm{c}}\right)\right] \\
& \varepsilon_{\mathrm{a}}=\varepsilon_{\mathrm{a}}{ }^{\prime}\left[1-j \tan \delta\left(\varepsilon_{\mathrm{a}}\right)\right] \\
& \varepsilon_{\mathrm{c}}=\varepsilon_{\mathrm{c}}{ }^{\prime}\left[1-j \tan \delta\left(\varepsilon_{\mathrm{c}}\right)\right]
\end{aligned}
$$

where $G_{\mathrm{a}}{ }^{\prime}, G_{\mathrm{c}}{ }^{\prime}, \varepsilon_{\mathrm{a}}{ }^{\prime}$, and $\varepsilon_{\mathrm{c}}{ }^{\prime}$ are real components of respective quantities and $\delta\left(\boldsymbol{G}_{\mathrm{a}}\right), \delta\left(\boldsymbol{G}_{\mathrm{c}}\right), \delta\left(\varepsilon_{\mathrm{a}}\right)$, and $\delta\left(\varepsilon_{\mathrm{c}}\right)$ are phase angles for each quantities.

If we neglect the higher terms than the square of $\tan \delta$, we obtain from (22) and (23),

$$
\begin{aligned}
e= & e_{\mathrm{c}} V_{\mathrm{c}} \frac{G_{\mathrm{a}}{ }^{\prime}}{G_{\mathrm{a}}{ }^{\prime}\left(1-K_{G}\right)+G_{\mathrm{c}}{ }^{\prime} K_{G}} \\
& \times\left[1+j \frac{G_{\mathrm{c}} K_{G}}{G_{\mathrm{a}}{ }^{\prime}\left(1-K_{G}\right)+G_{\mathrm{c}}{ }^{\prime} K_{G}}\right. \\
& \left.\times\left\{\tan \delta\left(G_{\mathrm{a}}\right)-\tan \delta\left(G_{\mathrm{c}}\right)\right\}\right] \\
& \times \frac{\varepsilon_{\mathrm{a}}{ }^{\prime}}{\varepsilon_{\mathrm{a}}{ }^{\prime}\left(1-K_{\varepsilon}\right)+\varepsilon_{\mathrm{c}}{ }^{\prime} K_{\varepsilon}}\left[1-j \frac{\varepsilon_{\mathrm{c}}{ }^{\prime} K_{\varepsilon}}{\varepsilon_{\mathrm{a}}{ }^{\prime}\left(1-K_{\varepsilon}\right)+\varepsilon_{\mathrm{c}}{ }^{\prime} K_{\varepsilon}}\right. \\
& \left.\times\left\{\tan \delta\left(\varepsilon_{\mathrm{a}}\right)-\tan \delta\left(\varepsilon_{\mathrm{c}}\right)\right\}\right] \\
d= & e_{\mathrm{c}} V_{\mathrm{c}} \frac{l}{G_{\mathrm{a}}{ }^{\prime}\left(1-L_{G}\right)+G_{\mathrm{c}}{ }^{\prime} L_{G}} \\
& \times\left[1-j \frac{G_{\mathrm{a}}{ }^{\prime}\left(1-L_{G}\right)}{G_{\mathrm{a}}{ }^{\prime}\left(1-L_{G}\right)+G_{\mathrm{c}}{ }^{\prime} L_{G}} \tan \delta\left(G_{\mathrm{a}}\right)\right.
\end{aligned}
$$

$$
\begin{aligned}
& \left.+j \frac{G_{\mathrm{c}}{ }^{\prime} L_{G}}{G_{\mathrm{a}}{ }^{\prime}\left(1-L_{G}\right)+G_{\mathrm{c}}{ }^{\prime} L_{G}} \tan \delta\left(G_{\mathrm{c}}\right)\right] \\
& \times \frac{\varepsilon_{\mathrm{a}}{ }^{\prime}}{\varepsilon_{\mathrm{a}}{ }^{\prime}\left(1-K_{\varepsilon}\right)+\varepsilon_{\mathrm{c}}{ }^{\prime} K_{\varepsilon}}\left[1-j \frac{\varepsilon_{\mathrm{c}}{ }^{\prime} K_{\varepsilon}}{\varepsilon_{\mathrm{a}}{ }^{\prime}\left(1-K_{\varepsilon}\right)+\varepsilon_{\mathrm{c}}{ }^{\prime} K_{\varepsilon}}\right. \\
& \left.\times\left\{\tan \delta\left(\varepsilon_{\mathrm{a}}\right)-\tan \delta\left(\varepsilon_{\mathrm{c}}\right)\right\}\right]
\end{aligned}
$$

If the relaxation takes place only in the nonpiezoelectric phase, then $\tan \delta\left(\boldsymbol{G}_{\mathrm{c}}\right)$ and $\tan \delta\left(\varepsilon_{\mathrm{c}}\right)$ can be put to zero in eq 28 and 29. If we define the $e$-constant and $d$-constant as follows:

$$
\begin{aligned}
& e=e^{\prime}-j e^{\prime \prime}=e^{\prime}\left(1-j \tan \delta_{e}\right) \\
& d=d^{\prime}-j d^{\prime \prime}=d^{\prime}\left(1-j \tan \delta_{d}\right)
\end{aligned}
$$

If there is no relaxation in the piezoelectric phase, $\tan \delta_{e}$ and $\tan \delta_{d}$ are given by

$$
\begin{aligned}
\tan \delta_{e}= & -\frac{G_{\mathrm{c}}{ }^{\prime} K_{G}}{G_{\mathrm{a}}{ }^{\prime}\left(1-K_{G}\right)+G_{\mathrm{c}}{ }^{\prime} K_{G}} \tan \delta\left(G_{\mathrm{a}}\right) \\
& +\frac{\varepsilon_{\mathrm{c}}{ }^{\prime} K_{\varepsilon}}{\varepsilon_{\mathrm{a}}{ }^{\prime}\left(1-K_{\varepsilon}\right)+\varepsilon_{\mathrm{c}}{ }^{\prime} K_{\mathrm{\varepsilon}}} \tan \delta\left(\varepsilon_{\mathrm{a}}\right) \\
\tan \delta_{d}= & +\frac{G_{\mathrm{a}}{ }^{\prime}\left(1-L_{G}\right)}{G_{\mathrm{a}}{ }^{\prime}\left(1-L_{G}\right)+G_{\mathrm{c}}{ }^{\prime} L_{G}} \tan \delta\left(G_{\mathrm{a}}\right) \\
& +\frac{\varepsilon_{\mathrm{c}}{ }^{\prime} K_{\mathrm{\varepsilon}}}{\varepsilon_{\mathrm{a}}{ }^{\prime}\left(1-K_{\varepsilon}\right)+\varepsilon_{\mathrm{c}}{ }^{\prime} K_{\varepsilon}} \tan \delta\left(\varepsilon_{\mathrm{a}}\right) .
\end{aligned}
$$

The difference of the sign of $\tan \delta\left(G_{a}\right)$ in eq 32 and 33 was first pointed out by Hayakawa and Wada. $^{3}$

\section{EXPERIMENTAL}

Silk fibers were oriented in a plane and tightly cemented together with either an aqueous solution of poly(vinyl alcohol) (PVA) or with an epoxy resin (Araldite). It is known that the silk fibers possess a piezoelectric constant with no marked relaxation and that PVA and epoxy resin display no piezoelectric effect.

Rectangular coordinates were assigned to the composite films: the $z$ axis in the direction of orientation of the fiber axis, the $y$ axis perpendicular to the film surface, and the $x$ axis in the film plane. As is usually observed in oriented biopolymers, shear stress in the film plane produces polarization normal to the film surface. Thus the values of $e_{25}$ and $d_{25}$ were determined.

The piezoelectric constants were measured with an apparatus newly designed in our laboratory, which determined the real and imaginary com- 
ponents of the $e$-constant, the $d$-constant and the elastic constant all at the same time. ${ }^{5}$ The polarization, stress, and strain were detected with a charge amplifier, a piezoelectric ceramics, and a strain gauge, respectively, under the condition of constant strain at a frequency of $10 \mathrm{~Hz}$.

A sinusoidal strain was imposed on the film in a direction at $45^{\circ}$ to the $z$ axis to obtain the values of $e_{25}$ and $d_{25}$. The elastic constants $E^{\prime}$ and $E^{\prime \prime}$ were also measured at the direction of $45^{\circ}$ to the direction of orientation of fiber axis.

Dielectric measurements were carried out by a capacitance bridge (General Radio Type 1615 A) at $1 \mathrm{KHz}$. The dielectric constant $\varepsilon$ was determined at the direction of the $y$ axis.

\section{RESULTS AND DISCUSSION}

In the present paper we will investigate the influence of the elastic and dielectric relaxations in the nonpiezoelectric amorphous phase on the measured values of the piezoelectric stressconstant, $e$, and strain-constant, $d$, of the polymer on the basis of some model experiments. We regard silk fibers as the piezoelectric crystalline phase and PVA or epoxy resin as the nonpiezoelectric amorphous phase.

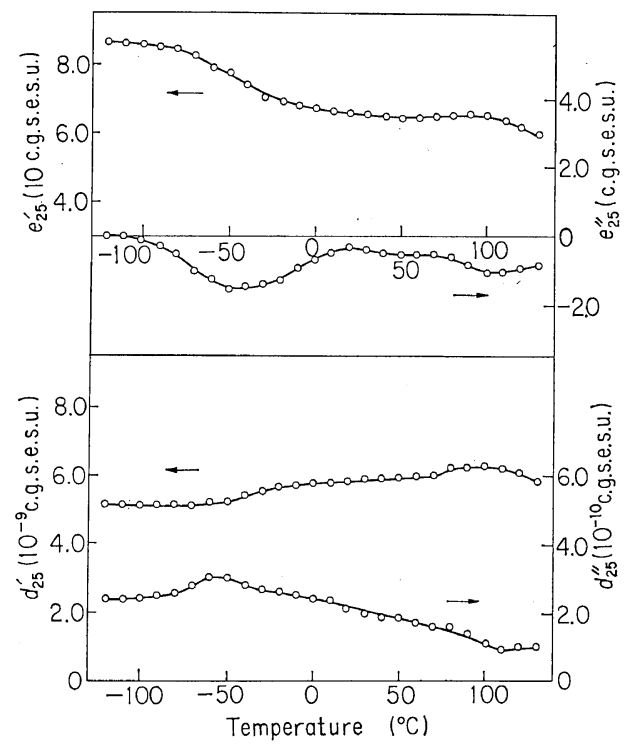

Figure 4. The temperature dependence of the piezoelectric stress-constant, $e_{25}$, and strain-constant, $d_{25}$, for the silk-epoxy resin system.
Figure 4 shows the temperature dependence of the $e$ - and $d$-constants respectively for the silkepoxy resin system. With increasing temperature $e_{25}^{\prime}$ decreases and $e_{25}^{\prime \prime}$ shows a maximum with a leading phase at about $-50^{\circ} \mathrm{C}$, wheres $d_{25}^{\prime}$ increases and $d_{25}^{\prime \prime}$ shows a maximum with a lagging phase at the same temperature. Both $e_{25}^{\prime}$ and $d_{25}^{\prime}$ decreases at temperatures higher than $100^{\circ} \mathrm{C}$.

The dielectric and elastic dispersions in epoxy resin have been determined for a film made of the resin and are shown in Figure 5. The dielectric loss $\varepsilon^{\prime \prime}$ measured at $1 \mathrm{kHz}$ shows a
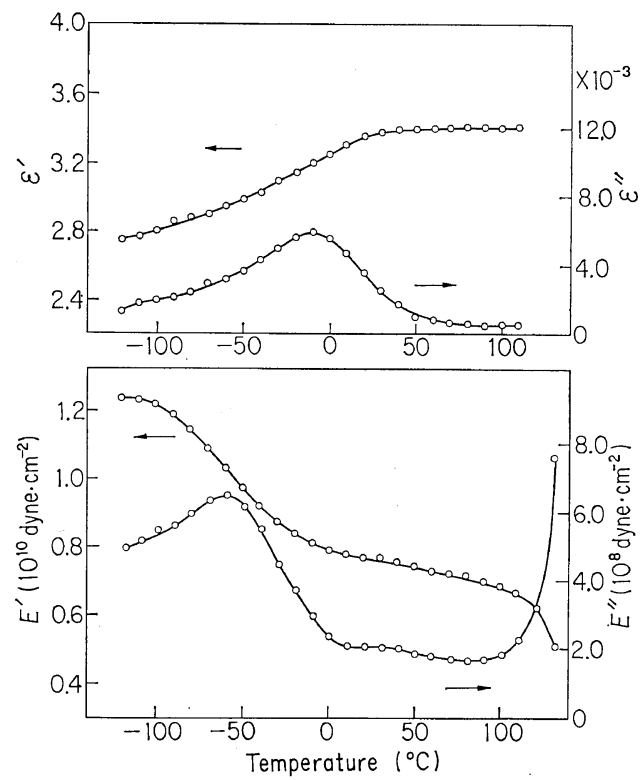

Figure 5. The temperature dispersion of the dielectric constant, $\varepsilon$, and the elastic constant, $E$, for the epoxy resin.

maximum at $-10^{\circ} \mathrm{C}$ and the maximum for the elastic loss $E^{\prime \prime}$ measured at $10 \mathrm{~Hz}$ appears at $-60^{\circ} \mathrm{C}$. Considering the difference in the measuring frequency, this relaxation in the epoxy resin should be responsible for piezoelectric relaxations in Figure 4.

In eq 22 and 23 we can see the influence of the variation of $G_{\mathrm{a}}$ on $e$ and $d$. The decrease of $G_{\mathrm{a}}$ should cause a decrease in $e^{\prime}$ accompanied with a maximum of $e^{\prime \prime}$ with a leading phase and the increase of $d^{\prime}$ accompanied with a maximum of $d^{\prime \prime}$ with a lagging phase.

The influence of the variation of $\varepsilon_{\mathrm{a}}$ on $e$ and 


\section{H. Yamagami and E. Fukada}

$d$ is also seen in eq 22 and 23. The increase of $\varepsilon_{\mathrm{a}}$ should cause an increase in both $e^{\prime}$ and $d^{\prime}$ accompanied with a maximum of $e^{\prime \prime}$ and $d^{\prime \prime}$ with a lagging phase.

When the temperature is increased, the decrease of $G_{\mathrm{a}}{ }^{\prime}$ and the increase of $\varepsilon_{\mathrm{a}}{ }^{\prime}$ take place at the same time. Consequently, as is seen in eq 22 and $23, G_{\mathrm{a}}$ and $\varepsilon_{\mathrm{a}}$ affect $e$ in opposite directions but $d$ in the same direction. The extent of the influence of $G_{\mathrm{a}}$ and $\varepsilon_{\mathrm{a}}$ with increasing temperature should depend on their respective relaxation strengths.

The maximum values of loss tangent of the four quantities for the silk-epoxy resin system are roughly $\tan \delta_{e}=-2 \times 10^{-2}, \tan \delta_{d}=6 \times 10^{-2}$, $\tan \delta_{E}=7 \times 10^{-2}$ and $\tan \delta_{\varepsilon}=2 \times 10^{-3}$. Equations 32 and 33 give the relations between these quantities, where $G_{\mathrm{a}}$ corresponds to $E$ and $\varepsilon_{\mathrm{a}}$ to $\varepsilon$. The magnitude of the dielectric loss $\tan \delta_{\varepsilon}$ is one order smaller than the elastic loss $\tan \delta_{E}$ and so the influence of dielectric relaxation can be ignored for the present case. Thus $\tan \delta_{e}$ and $\tan \delta_{d}$ are mainly determined by the value of $\tan \delta\left(\boldsymbol{G}_{\mathrm{a}}\right)$, which is $\tan \delta_{E}$ for the present.

We may therefore conclude that the relaxations

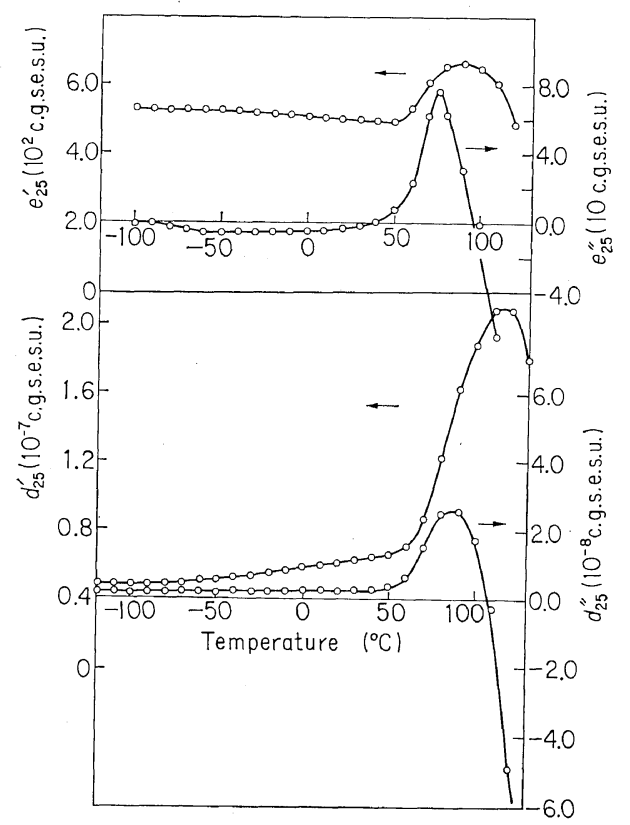

Figure 6. The temperature dependence of the piezoelectric stress-constant, $e_{25}$, and strain-constant, $d_{25}$, for the silk-poly(vinyl alcohol) system. in the $e$ - and $d$-constants for the silk-epoxy resin system are mainly due to the elastic relaxation in epoxy resin which adheres to the silk fibers. The decrease of $e_{25}^{\prime}$ and $d_{25}^{\prime}$ above $100^{\circ} \mathrm{C}$ seems to be caused by the increased conductivity in the system accompanied by the softening of the epoxy resin.

The temperature dependences of the $e$ - and $d$ constants for the silk-PVA system are shown in Figure 6. From $-100^{\circ} \mathrm{C}$ to $50^{\circ} \mathrm{C}, e_{25}^{\prime}$ slightly decreases with a leading phase and $d_{25}^{\prime}$ slightly increases with a lagging phase. This variation may reflect the gradual relaxation of the elastic constant of PVA, that is the influence of $G_{\mathrm{a}}$ similar to that in the silk-epoxy resin system. Above $50^{\circ} \mathrm{C}$ both $e_{25}^{\prime}$ and $d_{25}^{\prime}$ increase with increasing temperature, from $90^{\circ} \mathrm{C}$ to $110^{\circ} \mathrm{C}, e_{25}^{\prime}$ decreases and $d_{25}^{\prime}$ increases, and above $110^{\circ} \mathrm{C}$, both quantities decrease. The behavior above $50^{\circ} \mathrm{C}$ is different from that observed in the silkepoxy resin system.

Figure 7 shows the dielectric and elastic relaxations observed for PVA films which are made of the same material as used as the
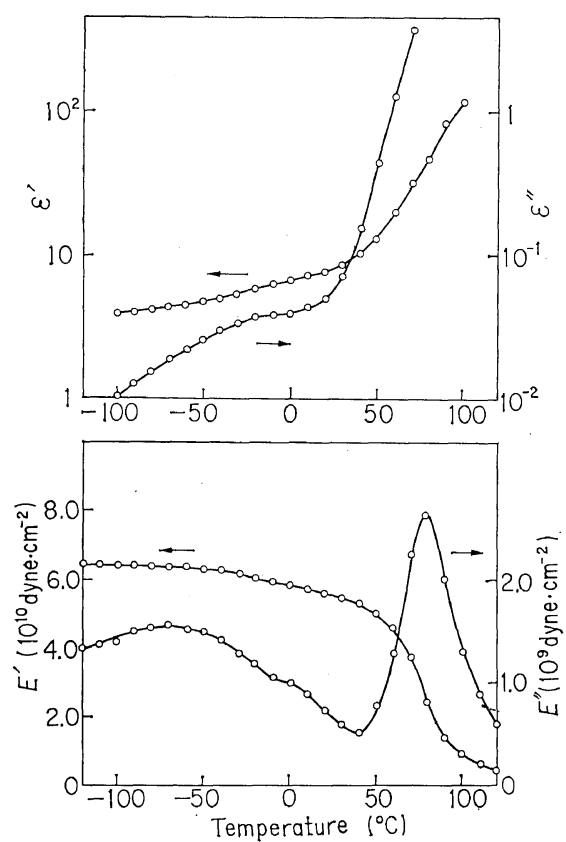

Figure 7. The temperature dispersion of the dielectric constant, $\varepsilon$, and the elastic constant, $E$, for the polyvinyl alcohol. 
adhesive. A very broad dispersion is seen for the elastic constants below $50^{\circ} \mathrm{C}$. The elastic constant at $10 \mathrm{~Hz}$ tends to decrease above $50^{\circ} \mathrm{C}$ and the elastic loss shows a peak at $80^{\circ} \mathrm{C}$. The dielectric constants $\varepsilon^{\prime}$ and $\varepsilon^{\prime \prime}$ measured at $1 \mathrm{KHz}$ also increase markedly above $50^{\circ} \mathrm{C}$. This relaxation above $50^{\circ} \mathrm{C}$ is due to the glass transition of PVA.

The values of maximum loss tangents at about $80^{\circ} \mathrm{C}$ are $\tan \delta_{e}=1.2 \times 10^{-1}, \tan \delta_{d}=1.7 \times 10^{-1}$, for the combined system and $\tan \delta_{E}=1 \times 10^{-1}$ and $\tan \delta_{\varepsilon}=2 \times 10^{-1}$ (at $80^{\circ} \mathrm{C}$ ) for PVA. The value of $\tan \delta_{\varepsilon}$ is larger than $\tan \delta_{E}$ and cannot be ignored. In eq 22 and 23 , the increase of $\varepsilon_{\mathrm{a}}$ causes the increase of both $e^{\prime}$ and $d^{\prime}$ accompanied with $\tan \delta_{e}$ and $\tan \delta_{d}$ with a lagging phase. Thus we may infer that the increase of $e_{25}^{\prime}$ and $d_{25}^{\prime}$ from $50^{\circ} \mathrm{C}$ to $90^{\circ} \mathrm{C}$ should be caused by the dielectric relaxation in the PVA phase. However, the elastic relaxation in PVA also takes place and its influence can be seen in the decrease of $e_{25}^{\prime}$ and the increase of $d_{25}^{\prime}$ from $90^{\circ} \mathrm{C}$ to $110^{\circ} \mathrm{C}$. This behavior is similar to that observed for the silk-epoxy resin system around $-50^{\circ} \mathrm{C}$. The decrease in both $e_{25}^{\prime}$ and $d_{25}^{\prime}$ above $110^{\circ} \mathrm{C}$ for the silk-PVA system should be caused by the increase of conductivity in the system.

\section{CONCLUSION}

We have descibed the results of the model experiments using two kinds of two phase polymer system. In the silk-epoxy resin system, the amount of dielectric relaxation in the epoxy resin system is smaller than the elastic relaxation at about $-50^{\circ} \mathrm{C}$. Then with increasing temperature the $e$-constant decreases and the $d$ constant increases for the system. This indicates the predominance of the influence of the elastic relaxation in the epoxy-resin phase.

In the silk-PVA system, the amount of dielectric relaxation in the PVA system is larger than the elastic relaxation above $50^{\circ} \mathrm{C}$. Then with increasing temperature, both $e$ - and $d$ constants increase for the system, indicating the predominance of the influence of the dielectric relaxation in the PVA phase.

This behavior in the silk-epoxy resin and silk-PVA systems can be well explained by eq. $22,23,32$, and 33 . In the ordinary piezoelectric polymers, the piezoelectric phase consists of the crystalline regions and well oriented regions of molecules and the nonpiezoelectic phase consists of the amorphous regions. If the relaxation in the intrinsic piezoelectric constants in the piezoelectric phase can be ignored, the observed piezoelectric relaxation can be ascribed to the elastic and dielectric relaxations in the amorphous phase surrounding the piezoelectric phase. In principle both the elastic and dielectric relaxations in the amorphous phase have an effect on the piezoelectric constant at the same time. However, in actual measurements the predominance of either kind is often observed, which may be caused by the difference in relaxation strengths and relaxation times in elastic and dielectric properties of polymers.

Acknowledgement. The authors wish to express their appreciation to Mr. M. Date and Mr. T. Furukawa for their helpful discussion. This work was supported by a research grant for the Studies on Life Sciences in the Institute of Physical and Chemical Research.

\section{REFERENCES}

1. E. Fukada, Progr. Polym. Sci. Japan, 2, 329 (1971).

2. R. Hayakawa and Y. Wada, Advan. Polym. Sci., 11, 1 (1973).

3. R. Hayakawa and Y. Wada, Rep. Progr. Polym. Phys. Japan, 15, 369, 373 (1972).

4. M. Date, ibid., 15, 385 (1972).

5. T. Furukawa and E. Fukada, ibid., 16, 457 (1973). 